\title{
CME Rheumatology
}

Edited by Prof A Gerry Wilson, professor of rheumatology and academic director of specialised medicine, Department of Infection and Immunity, The University of Sheffield Medical School, Sheffield, UK; Dr Rachel S Tattersall, consultant in adult and adolescent rheumatology, Royal Hallamshire Hospital, and Sheffield Children's Hospital, Sheffield, UK

\section{The 'therapeutic}

\section{window' and treating to target in rheumatoid arthritis}

\author{
Laura Hunt, ${ }^{1,2}$ clinical research fellow and \\ rheumatology specialist registrar; \\ Maya Buch, 1,2 NIHR clinician specialist, \\ senior lecturer and honorary consultant \\ rheumatologist \\ ${ }^{1}$ Leeds Institute of Rheumatic and \\ Musculoskeletal Medicine, University of \\ Leeds, Leeds, UK; \\ ${ }^{2}$ NIHR Leeds Musculoskeletal Biomedical \\ Research Unit, Leeds Teaching Hospitals \\ NHS Trust, Leeds, UK
}

Rheumatoid arthritis (RA) is an autoimmune condition characterised by a chronic inflammatory arthritis. If inadequately treated it results in joint destruction with subsequent deformity, disability and substantial socio-economic costs. RA affects $0.5-1 \%$ of the population in the UK and healthcare costs reach an estimated

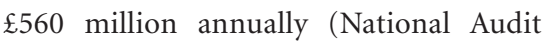
Office). Fortunately, the overall prognosis for RA has improved dramatically in recent years owing to innovative research and advances in available therapies.

Historically, non-steroidal antiinflammatory drugs (NSAIDs) were the mainstay of treatment, followed by glucocorticoids and finally disease-modifying anti-rheumatic drugs (DMARDS) such as gold, sulphasalazine and penicillamine. Although this treatment strategy managed the symptoms of RA, delay in initiation of DMARDS resulted in persistent inflammation with subsequent joint destruction and long-term disability. A different approach was therefore imperative.

This article will focus on the concept of a therapeutic window for managing RA and the treatment strategies now recommended, with an indication of how the treatment paradigm continues to evolve.

\section{Therapeutic window in rheumatoid arthritis}

Since the 1990s rheumatologists have been exploring the concept of a 'window of opportunity' in the treatment of RA. ${ }^{1}$ To explain this concept, comparisons can be drawn from other diseases such as cancer, where treating the disease from the onset equates to less disease burden and leads to maximum effect of therapy. In RA this translates to suppressing the inflammation before the irreversible joint damage occurs. Evidence to support this theory in RA is derived from studies which have demonstrated that early intervention results in optimal clinical outcomes. ${ }^{2-4}$ This has been further validated by radiographic data illustrating that early treatment equates to less erosive disease. ${ }^{3,5}$

\section{Principles to treating rheumatoid arthritis}

The recognition of a 'window of opportunity' has led to a paradigm shift in the management of RA. DMARDS, in particular methotrexate, now form the cornerstone of treatment. Therapies are frequently 'stepped up' and used in combination to achieve disease control. Pivotal trials have provided insight into drug efficacy and treatment strategies, culminating in key principles for the modern management of RA.

\section{Aim for early diagnosis}

We know from longitudinal studies that functional capacity in patients with RA deteriorates with time. ${ }^{6}$ Long-term disability is primarily caused by joint damage that occurs

Box 1. Target population: patients who have at least one joint with definite clinical synovitis (swelling), not better explained by another disease, should be tested. ${ }^{9}$

\section{A. Joint involvement}

1 large joint

2-10 large joints

1-3 small joints (with or without involvement of large joints)

4-10 small joints (with or without involvement of large joints)

$>10$ joints (at least 1 small joint)

B. Serology (at least one test result is needed for classification) Negative RF and negative ACPA Low positive RF or low positive ACPA High positive RF or high positive ACPA

C. Acute-phase reactants (at least one test result is needed for classification) Normal CRP and normal ESR

\section{Duration of symptoms}

$<6$ weeks

$>6$ weeks

$\mathrm{ACPA}=$ anti-citrullinated protein antibodies; $\mathrm{CRP}=\mathrm{C}$-reactive protein; $\mathrm{ESR}=$ erythrocyte sedimentation rate; $\mathrm{RA}=$ rheumatoid arthritis; $\mathrm{RF}=$ rheumatoid factor.

Classification criteria for RA (score-based algorithm: add score of categories A-D; a score of $\geq 6 / 10$ is needed for classification of a patient as having definite RA). 
subsequent to ongoing inflammation and disease activity. In early RA with high disease activity it is possible to detect changes in joint damage within 3 months using plain radiographs. $^{7}$ Therefore, early diagnosis and immediate intervention with diseasemodifying therapy is vital to prevent disability. The heterogeneity in presentation of RA can lead to delays in diagnosis, and previous criteria for RA have demonstrated low sensitivity and specificity for early disease. ${ }^{8}$ In recognition of this, the American College of Rheumatology (ACR) and European League Against Rheumatism (EULAR) collaborated in 2010 to define a new classification criteria for RA to allow diagnosis at the earliest possible stage (Box 1). ${ }^{9}$ Referral to a rheumatologist by general practitioners and other physicians is recommended when there is any clinical suspicion (symptoms +/- signs) of an inflammatory arthritis. ${ }^{10}$ This new criteria combined with prompt referral is paramount in preventing the undesirable sequelae of RA.

\section{Early initiation of therapy}

In clinical practice, the concept of a therapeutic window in RA involves the immediate initiation of therapy upon diagnosis, with minimum delay. There is unequivocal evidence to support the early commencement of DMARDs. Individuals assessed and
Box 2. Ten recommendations on treating rheumatoid arthritis to target based on both evidence and expert opinion. ${ }^{22}$

1 The primary target for treatment of RA should be a state of clinical remission.

2 Clinical remission is defined as the absence of signs and symptoms of significant inflammatory disease activity.

3 While remission should be a clear target, based on available evidence low disease activity may be an acceptable alternative therapeutic goal, particularly in established long-standing disease.

4 Until the desired treatment target is reached, drug therapy should be adjusted at least every 3 months.

5 Measures of disease activity must be obtained and documented regularly, as frequently as monthly for patients with high/moderate disease activity or less frequently (such as every 3-6 months) for patients in sustained low disease activity or remission.

6 The use of validated composite measures of disease activity, which include joint assessments, is needed in routine clinical practice to guide treatment decisions.

7 Structural changes and functional impairment should be considered when making clinical decisions, in addition to assessing composite measures of disease activity.

8 The desired treatment target should be maintained throughout the remaining course of the disease.

9 The choice of the (composite) measure of disease activity and the level of the target value may be influenced by consideration of comorbidities, patient factors and drug-related risks.

10 The patient has to be appropriately informed about the treatment target and the strategy planned to reach this target under the supervision of the rheumatologist.

$\mathrm{RA}=$ rheumatoid arthritis.

\section{Key points}

Rheumatoid arthritis (RA) can lead to significant long-term morbidity and mortality

It is important to aim for early diagnosis to limit the structural damage that occurs with prolonged inflammation

Commencing disease-modifying anti-rheumatic drugs (DMARD) therapy and glucocorticoids as early as possible and titrating therapy as appropriate improves clinical outcomes

Patients must be closely monitored for inflammatory disease with regular clinical assessments and treated to a target of clinical remission

If low disease activity or clinical remission cannot be achieved with standard DMARDs biological therapies can be initiated

KEY WORDS: Rheumatoid arthritis, treatment, therapeutic window, disease modifying agents, biological therapy treated with disease duration of $<12$ weeks have been shown to have better clinical outcomes and less radiographic progression than those presenting with longer disease duration. ${ }^{2}$ A meta-analysis of 14 randomised control trials confirmed early initiation of therapy in individuals with symptoms of less than a year corresponded to better response to DMARDs. ${ }^{11}$ In fact, shorter disease duration was the strongest variable associated with improved clinical response. There is a wealth of clinical trial data supporting this approach. ${ }^{12-16}$

\section{Treat to a target}

Treating to a predefined target is undertaken in several pathologies to enable physicians and patients to discuss and adopt therapeutic changes within distinct time frames. Improved clinical outcomes in hypertension and diabetes have been witnessed subsequent to close monitoring and predefined targets in blood pressure and HbAlc levels, respectively. In RA the development of reliable composite measures to assess disease activity, such as the Disease Activity Score 28 (DAS28), ${ }^{17}$ has allowed the principles of treating to a target to be applied to RA management. Clinical remission - the absence of inflammatory disease - is accepted as the target of treatment of RA. In those individuals where this is not possible, a state of low disease activity is accepted. Attainment of this goal has been demonstrated to halt joint damage and hence improve long-term clinical outcomes. ${ }^{14,18}$

\section{Monitoring disease and optimising therapy}

In order to achieve a treatment target of remission or low disease activity, a structured patient management approach is required. This is supported by trials that compared routine outpatient review vs intensive follow up (defined as frequent visits with prompt titration of drug therapy). Those receiving intensive follow up had significant improvement in clinical outcome, physical function and structural damage. ${ }^{15,16,19,20}$ Patients treated early and aggressively with DMARDs also had reduced work disability, sick leave and premature retirement rates. The aim is to achieve the 
predefined target, ideally within 3 months, but at least within 6 months of diagnosis. The interval between assessments should be at least 3 months, but monthly visits may be necessary until a period of sustained low disease activity or remission is achieved. It appears that irrespective of treatment therapy, treating to a target, regular follow up and monitoring of disease activity with responsive drug escalation all lead to sustained clinical and functional benefit. ${ }^{21}$ The principles described above form the foundations of guidelines recently published for the management of RA. 22 'Treat to target' is an international initiative developed from a systematic review of all strategy trials in RA and expert opinion (Box 2). It advocates early diagnosis, immediate implementation of disease modifying therapy, monitoring of disease activity and the optimisation of therapy accordingly.

\section{Treatment strategies}

Recommendations for pharmacological therapies in RA have been developed by the National Institute for Health and Care Excellence (NICE) and EULAR. ${ }^{23,24}$ The clinical efficacy data and safety profile of methotrexate endorse it as the anchor drug of choice. Alternative DMARDs are available for those individuals intolerant of methotrexate. DMARDs can also be added in combination as appropriate. Glucocorticoids are recommended as an adjuvant therapy at disease onset due to their disease-modifying and anti-inflammatory properties. ${ }^{19}$ Clinical trials have consistently demonstrated that tight disease control with appropriate escalation of therapy is fundamental in achieving low disease activity. ${ }^{16,20}$ Therefore, when synthetic DMARDs fail, escalation to one of the biological therapies is advised (Table 1).

Biological therapies have revolutionised disease outcomes in RA. In active, longstanding disease with an inadequate response to methotrexate the addition of a biological agent has significantly improved clinical and structural outcomes. ${ }^{25}$ Evaluation of biologicals when initiated early suggests more optimal suppression of inflammation may be achieved, reinforcing the concept of a 'window of opportunity'. When tumour necrosis factor inhibitors were initiated as
Table 1. Biological therapies approved by NICE for the treatment of rheumatoid arthritis.

Route of administration

NICE approval

Tumour necrosis factor

inhibitors

- Adalimumab

Fortnightly, subcutaneous injection

First line biological therapy

- Certolizumab Pegol

Fortnightly, subcutaneous injection

- Etanercept

Weekly, subcutaneous injection

- Golimumab

Monthly, subcutaneous injection

- Infliximab

8-weekly, intravenous infusion

IL-6 inhibitors

- Tocilizumab

Monthly, intravenous infusion

First line biological therapy

B cell targeted therapy

- Rituximab

Intravenous infusion, two doses administered over 2 weeks at disease flare. Minimum duration between therapy 6 months

T cell targeted therapy

- Abatacept

Monthly, intravenous infusion

Second line biological therapy first line treatment in early RA, higher rates of low disease activity and remission were achieved. ${ }^{20,26}$ In the UK, NICE stipulates that treatment with methotrexate and one other DMARD is necessary prior to escalation. With this is mind it is imperative that treatment is regularly reviewed and titrated in order to allow appropriate escalation when standard therapy is not sufficient.

\section{The future of rheumatoid arthritis}

Research and clinical experience have provided rheumatologists with effective therapies and strategies for managing RA. However, the optimum treatment pathway has not yet been established. While a good proportion of patients may achieve clinical remission with synthetic DMARDS alone (particularly with a treatment to target approach), it remains unclear whether an initial period of aggressive therapy with biological agents would equate to improved clinical outcomes. The possibility of drug-free remission and additional advantages of therapy such as cardiovascular benefits remain areas for further evaluation that will help refine treatment approaches in the future.

It is also important to monitor for structural damage in addition to disease activity. Patients in clinical remission have been observed to continue to show structural progression, attributed to persistent subclinical synovitis. ${ }^{27,28}$ This has partly driven evaluation of more accurate methods of defining and monitoring disease. Ultrasound has emerged as a useful and practical tool in detecting subclinical inflammation that is not identified clinically. Similarly, advances in the understanding and methods of analysis of immune dysregulation provide additional disease markers. Targets for remission in subsequent years could include achieving imaging and immunological remission.

The potential for curing RA is a realistic goal in light of the novel drug therapies in development. As our understanding of the pathogenesis of RA expands, the prospect for prevention is also on the horizon. The last 20 years have seen dramatic changes in the way we approach RA; however, the future may hold even greater promise.

\section{References}

1 Quinn MA, Emery P. Window of opportunity in early rheumatoid arthritis: possibility of altering the disease process with early intervention. Clin Exp Rheumatol 2003;21:S154-7.

2 Nell VP, Machold KP, Eberl G et al. Benefit of very early referral and very early therapy with disease-modifying anti-rheumatic drugs in 
patients with early rheumatoid arthritis. Rheumatology (Oxford) 2004;43:906-14.

3 van der Linden MP, le Cessie S, Raza K et al. Long-term impact of delay in assessment of patients with early arthritis. Arthritis Rheum 2010;62:3537-46.

4 Lard LR, Visser H, Speyer I et al. Early versus delayed treatment in patients with recentonset rheumatoid arthritis: comparison of two cohorts who received different treatment strategies. Am J Med 2001;111:446-51.

5 Finckh A, Liang MH, van Herckenrode CM, de Pablo P. Long-term impact of early treatment on radiographic progression in rheumatoid arthritis: A meta-analysis. Arthritis Rheum 2006;55:864-72.

6 Yelin E, Trupin L, Wong B, Rush S. The impact of functional status and change in functional status on mortality over 18 years among persons with rheumatoid arthritis. $J$ Rheumatol 2002;29:1851-7.

7 Bruynesteyn K, Landewé R, van der Linden S, van der Heijde D. Radiography as primary outcome in rheumatoid arthritis: acceptable sample sizes for trials with 3 months' follow up. Ann Rheum Dis 2004;63:1413-8.

8 Banal F, Dougados M, Combescure C, Gossec L. Sensitivity and specificity of the American College of Rheumatology 1987 criteria for the diagnosis of rheumatoid arthritis according to disease duration: a systematic literature review and metaanalysis. Ann Rheum Dis 2009;68:1184-91.

9 Aletaha D, Neogi T, Silman AJ et al. 2010 Rheumatoid arthritis classification criteria: an American College of Rheumatology/ European League Against Rheumatism collaborative initiative. Arthritis Rheum 2010;62:2569-81.

10 Emery P, Breedveld FC, Dougados M et al. Early referral recommendation for newly diagnosed rheumatoid arthritis: evidence based development of a clinical guide. Ann Rheum Dis 2002;61:290-7.

11 Anderson JJ, Wells G, Verhoeven AC, Felson DT. Factors predicting response to treatment in rheumatoid arthritis: the importance of disease duration. Arthritis Rheum 2000;43:22-9.

12 O'Dell JR, Haire CE, Erikson N et al. Treatment of rheumatoid arthritis with methotrexate alone, sulfasalazine and hydroxychloroquine, or a combination of all three medications. $N$ Engl J Med 1996;334:1287-91.

13 Boers M, Verhoeven AC, Markusse HM et al. Randomised comparison of combined step-down prednisolone, methotrexate and sulphasalazine with sulphasalazine alone in early rheumatoid arthritis. Lancet 1997;350:309-18.

14 Rantalaiho V, Korpela M, Laasonen L et al. Early combination disease-modifying antirheumatic drug therapy and tight disease control improve long-term radiologic outcome in patients with early rheumatoid arthritis: the 11-year results of the Finnish Rheumatoid Arthritis Combination Therapy trial. Arthritis Res Ther 2010;12:R122.

15 Grigor C, Capell H, Stirling A et al. Effect of a treatment strategy of tight control for rheumatoid arthritis (the TICORA study): a single-blind randomised controlled trial. Lancet 2004;364:263-9.

16 Verstappen SM, Jacobs JW, van der Veen $\mathrm{MJ}$ et al. Intensive treatment with methotrexate in early rheumatoid arthritis: aiming for remission. Computer Assisted Management in Early Rheumatoid Arthritis (CAMERA, an open-label strategy trial). Ann Rheum Dis 2007;66:1443-9.

17 Aletaha D, Smolen JS. The definition and measurement of disease modification in inflammatory rheumatic diseases. Rheum Dis Clin North Am 2006;32:9-44.

18 Smolen JS, Han C, van der Heijde DM et al. Radiographic changes in rheumatoid arthritis patients attaining different disease activity states with methotrexate monotherapy and infliximab plus methotrexate: the impacts of remission and tumour necrosis factor blockade. Ann Rheum Dis 2009;68:823-7.

19 Bakker MF, Jacobs JW, Welsing PM et al. Low-dose prednisone inclusion in a methotrexate-based, tight control strategy for early rheumatoid arthritis: a randomized trial. Ann Intern Med 2012;156:329-39.

20 Goekoop-Ruiterman YP, de Vries-Bouwstra JK, Allaart CF et al. Comparison of treatment strategies in early rheumatoid arthritis: a randomized trial. Ann Intern Med 2007;146:406-15.

21 Klarenbeek NB, Güler-Yüksel M, van der Kooji SM et al. The impact of four dynamic, goal-steered treatment strategies on the 5-year outcomes of rheumatoid arthritis patients in the BeSt study. Ann Rheum Dis 2011;70:1039-46.

22 Smolen JS, Aletaha D, Bijlsma JW et al. Treating rheumatoid arthritis to target: recommendations of an international task force. Ann Rheum Dis 2010;69:631-7.

23 Smolen JS, Landewé R, Breedveld FC et al. EULAR recommendations for the management of rheumatoid arthritis with synthetic and biological disease-modifying antirheumatic drugs. Ann Rheum Dis 2010;69:964-75.

24 National Institute for Health and Care Excellence. Rheumatoid arthritis: The management of rheumatoid arthritis in adults. London: NICE, 2009. www.nice.org.uk/ nicemedia/pdf/CG79NICEGuideline.pdf [Accessed 30 May 2013].

25 van der Heijde D, Klareskog L, RodriguezValverde $\mathrm{V}$ et al. Comparison of etanercept and methotrexate, alone and combined, in the treatment of rheumatoid arthritis: two-year clinical and radiographic results from the TEMPO study, a double-blind, randomized trial. Arthritis Rheum 2006;54:1063-74.

26 Emery P, Breedveld FC, Hall S et al. Comparison of methotrexate monotherapy with a combination of methotrexate and etanercept in active, early, moderate to severe rheumatoid arthritis (COMET): a randomised, double-blind, parallel treatment trial. Lancet 2008;372:375-82.

27 Molenaar ET, Voskuyl AE, Dinant HJ et al. Progression of radiologic damage in patients with rheumatoid arthritis in clinical remission. Arthritis Rheum 2004;50:36-42.

28 Brown AK, Quinn MA, Karim Z et al. Presence of significant synovitis in rheumatoid arthritis patients with disease-modifying antirheumatic drug-induced clinical remission: evidence from an imaging study may explain structural progression. Arthritis Rheum 2006;54:3761-73.

Address for correspondence: $\operatorname{Dr} M$ Buch, Leeds Institute of Rheumatic and Musculoskeletal Medicine, University of Leeds, Chapel Allerton Hospital, Chapeltown Road, Leeds LS7 4SA. Email: M.Buch@leeds.ac.uk 